Pacific

Journal of

Mathematics

AN ENDPOINT ESTIMATE FOR SOME MAXIMAL OPERATORS ASSOCIATED TO SUBMANIFOLDS OF LOW CODIMENSION

YA RYONG HeO 


\title{
AN ENDPOINT ESTIMATE FOR SOME MAXIMAL OPERATORS ASSOCIATED TO SUBMANIFOLDS OF LOW CODIMENSION
}

\author{
YA RYONG HEO
}

We show that the maximal operator

$$
\mathcal{M} f(x)=\sup _{j \in \mathbb{Z}}\left|\int_{\mathbb{R}^{d}} f\left(x-2^{j} y\right) d \mu(y)\right|
$$

maps $H^{1}$ into $L^{1, \infty}$ under certain assumptions on the decay of $\hat{\mu}$ and the geometry of $\operatorname{supp}(\mu)$.

\section{Introduction and statement of results.}

In this paper we consider the lacunary maximal operator $\mathcal{M}$ defined by

$$
\mathcal{M} f(x)=\sup _{j \in \mathbb{Z}}\left|\int_{\mathbb{R}^{d}} f\left(x-2^{j} y\right) d \mu(y)\right| .
$$

Here $d \geq 1$ is an integer. When $\mu$ is a finite positive Borel measure on $\mathbb{R}^{d}$, it is proved in $[\mathbf{D R}]$ that if the Fourier transform of $\mu$ satisfies

$$
|\hat{\mu}(\xi)| \leq c(1+|\xi|)^{-\alpha}
$$

for some $\alpha>0$, then (1) is bounded on $L^{p}\left(\mathbb{R}^{d}\right)$ for $1<p \leq \infty$. Also when $\alpha=\frac{d}{2}$, it is proved in $[\mathbf{O}]$ that $(1)$ maps $H^{1}\left(\mathbb{R}^{d}\right)$ into $L^{1, \infty}\left(\mathbb{R}^{d}\right)$. Here $H^{1}$ denotes the usual real-variable Hardy space. On the other hand, Theorem 4 in [C2] states that if $\mu$ is the Lebesque measure $\sigma_{d-1}$ on the unit sphere $\sum_{d-1}$ in $\mathbb{R}^{d}$ then (1) maps $H^{1}\left(\mathbb{R}^{d}\right)$ into $L^{1, \infty}\left(\mathbb{R}^{d}\right)$. The purpose of this paper is to prove a result which includes the results in [O] and Theorem 4 in [C2] as special cases and which also applies to maximal operators associated to some submanifolds of codimension greater than 1 . The method of proof is an adaptation of the argument in $[\mathbf{O}]$, which is based on the basic approach in $[\mathbf{C 2}]$.

For each bounded subset $A$ of $\mathbb{R}^{d}$ and $0<\epsilon<1$, define $N(A, \epsilon)$ as the smallest number of $\epsilon$-balls needed to cover $A$, i.e.,

$$
N(A, \epsilon)=\min \left\{m: A \subset \bigcup_{i=1}^{m} B\left(x_{i}, \epsilon\right) \text { for some } x_{i} \in \mathbb{R}^{d}\right\} .
$$

Now we state our main result. 
Theorem 1. Suppose $\mu$ is a finite positive Borel measure on $\mathbb{R}^{d}$ with compact support such that for $0<\epsilon<1$

$$
N(\operatorname{supp}(\mu), \epsilon) \leq c \epsilon^{-n}, \quad|\hat{\mu}(\xi)| \leq c(1+|\xi|)^{-\frac{n}{2}}
$$

then (1) maps $H^{1}\left(\mathbb{R}^{d}\right)$ into $L^{1, \infty}\left(\mathbb{R}^{d}\right)$ when $0<n \leq d$.

In particular if $n=d$, then we obtain the result of $[\mathbf{O}]$. Moreover we have the following.

Corollary 2. Suppose $M \subset \mathbb{R}^{d}$ is a $C^{1}$ submanifold of dimension n equipped with a finite positive Borel measure $\mu$ which has compact support. If the Fourier transform of $\mu$ satisfies the decay estimate

$$
|\hat{\mu}(\xi)| \leq c(1+|\xi|)^{-\frac{n}{2}}
$$

then (1) maps $H^{1}\left(\mathbb{R}^{d}\right)$ into $L^{1, \infty}\left(\mathbb{R}^{d}\right)$ when $0<n \leq d$.

Proof. Let $A$ be a bounded subset of $\mathbb{R}^{n}$ and $f: A \mapsto \mathbb{R}^{d}$ be a Lipschitz map. Then it is easy to show that

$$
N(f(A), \epsilon) \leq c N(A, \epsilon) \leq c \epsilon^{-n} .
$$

If $M$ is a $C^{1}$ submanifold of $\mathbb{R}^{d}$, then we can view $M$ locally as the graph of a vector-valued $C^{1}$ function defined on its tangent plane. Hence by (3) and compactness of $\operatorname{supp}(\mu)$, we have $N(\operatorname{supp}(\mu), \epsilon) \leq c \epsilon^{-n}$. By applying Theorem 1, we obtain the conclusion.

In particular if $M$ is $\sum_{d-1}$ and $\mu$ is $\sigma_{d-1}$, then we obtain Theorem 4 in $[\mathbf{C 2}]$. Also, as was treated in $[\mathbf{C D M M}]$ and $[\mathbf{C M}]$, if $M$ is a smooth compact convex hypersurface of finite type in $\mathbb{R}^{1+n}$, with Gaussian curvature $\kappa$ and surface measure $\mu$, then the Fourier transform $\widehat{\kappa^{1 / 2} \mu}(\xi)$ decays as $|\xi|^{-\frac{n}{2}}$ as $|\xi|$ goes to infinity. Hence Corollary 2 holds for $\kappa^{1 / 2} \mu$ when $n \geq 1$.

Our proof follows the methods of $[\mathbf{C 2}]$ and $[\mathbf{O}]$. What is different from $[\mathbf{O}]$ is the use of the geometry of $\operatorname{supp}(\mu)$. We use the geometry of $\operatorname{supp}(\mu)$ in proving Lemma 5 . The use of geometry of $\operatorname{supp}(\mu)$ allows us to put a weaker decay condition on $\widehat{\mu}$. Littman $[\mathbf{L}]$ showed that, if $M \subset \mathbb{R}^{1+n}$ is a smooth submanifold of dimension $n$ and has at least $l$ nonzero principal curvatures everywhere on $\operatorname{supp}(\mu)$, where $\mu$ is smooth and compactly supported, then

$$
|\hat{\mu}(\xi)| \leq c(1+|\xi|)^{-\frac{l}{2}} .
$$

Hence when $l=n \geq 1$, Corollary 2 can be applied.

As was indicated in [C3], the proof of Littman's theorem goes unchanged to establish the following. Suppose that $M \subset \mathbb{R}^{d}$ is a smooth manifold of dimension $n$, and $\mu$ is a smooth compactly supported measure on $M$. For fixed $b \in M$, we can view $M$ locally as a graph of a vector-valued function 
$\psi(x)$ defined on its tangent plane. Let $N_{b}(M)$ be a collection of a unit vector normal to $M$ at $b$ then for each $v \in N_{b}(M)$ the function $\langle\psi(x), v\rangle$ has a critical point at $x=b$. Suppose that for all $b \in M$ in some neighborhood of $\operatorname{supp}(\mu)$ and for all $v \in N_{b}(M)$ we have

$$
\left.\operatorname{det} \mathrm{D}^{2}\langle\psi(x), v\rangle\right|_{x=b} \neq 0 \text {. }
$$

Then

$$
|\hat{\mu}(\xi)| \leq c(1+|\xi|)^{-\frac{n}{2}}
$$

Hence Corollary 2 can be applied in this case also. The condition (4) is controlled by the second-order terms in the Taylor expansion of $\psi$ at $b$. We give some examples which satisfy (5).

\section{Example 3.}

(3.1) For $n=2 m$ and $d=n+2$, let $x, y \in \mathbb{R}^{m}$ and $M$ be the manifold described by $\left(x, y ;|x|^{2}-|y|^{2}, x \cdot y\right)$, then a smooth measure $\mu$ supported in a sufficiently small neighborhood of the origin satisfies (5) when $m \geq 1$. So Corollary 2 holds for this $\mu$ when $m \geq 1$.

(3.2) For $n=4 m$ and $d=n+2$, let $x, y, z, u \in \mathbb{R}^{m}$ and $M$ be the manifold described by $(x, y, z, u ; x \cdot z+y \cdot u, x \cdot u-y \cdot z)$, then a smooth measure $\mu$ supported in a sufficiently small neighborhood of the origin satisfies (5) when $m \geq 1$. So Corollary 2 holds for this $\mu$ when $m \geq 1$.

(3.3) For $n=4 m$ and $d=n+3$, let $x, y, z, u \in \mathbb{R}^{m}$ and $M$ be the manifold described by $\left(x, y, z, u ;|x|^{2}-|y|^{2}-|z|^{2}+|u|^{2}, x \cdot y-z \cdot u, x \cdot z+y \cdot u\right)$, then a smooth measure $\mu$ supported in a sufficiently small neighborhood of the origin satisfies (5) when $m \geq 1$. So Corollary 2 holds for this $\mu$ when $m \geq 1$.

\section{Preliminaries.}

Notation. If $Q$ is a dyadic cube in $\mathbb{R}^{d}$ with side-length $2^{j}$, we write $\sigma(Q)=$ $j$. For $\sigma \in \mathbb{Z}, \Re_{\sigma}$ denotes the collection of dyadic cubes $Q \in \mathbb{R}^{d}$ with $\sigma(Q)=\sigma$. And for $Q \in \Re_{\sigma}, Q^{*}$ denotes $Q+\left[-2^{\sigma}, 2^{\sigma}\right]^{d} \cdot|\cdot|$ denotes the Lebesgue measure.

The following Lemma is taken from [O] (see Lemma 1).

Lemma 4. Suppose $\alpha>0$ is given, and given any finite collection of dyadic cubes $\{Q\}_{Q \in \mathcal{C}}$ in $\mathbb{R}^{d}$, and corresponding collection of positive numbers $\left\{\lambda_{Q}\right\}_{Q \in \mathcal{C}}$ there exists a finite collection of pairwise disjoint dyadic cubes $\{S\}_{S \in \mathcal{S}}$ such that each $Q \in \mathcal{C}$ is contained for some $S \in \mathcal{S}$ and

(4.1) $\sum_{Q \subset S} \lambda_{Q} \leq 3^{d} \alpha|S|$

(4.2) $\sum_{S \in \mathcal{S}}|S| \leq \frac{1}{\alpha} \sum \lambda_{Q}$

$$
\left\|\sum_{\substack{\text { Q: not contained } \\ \text { in any S }}} \lambda_{Q}|Q|^{-1} \chi_{Q}\right\|_{L^{\infty}} \leq \alpha \text {. }
$$


Lemma 5 (cf. [C2, Lemma 5.1]). Suppose given the following: $0<n \leq d$, a Borel measure $\mu$ defined on a compact subset of $\mathbb{R}^{d}$ with $N(\operatorname{supp}(\mu), \epsilon) \leq$ $c \epsilon^{-n}$ for $0<\epsilon<1$, some $\alpha>0$, a finite collection $\mathcal{S}$ of pairwise disjoint dyadic cubes $S \subset \mathbb{R}^{d}$, a finite collection $\mathcal{C}$ of dyadic cubes $Q \subset \mathbb{R}^{d}$ such that each $Q \in \mathcal{C}$ is contained in some $S=S(Q) \in \mathcal{S}$ and for each $Q \in \mathcal{C}$ a positive number $\lambda_{Q}$ is assigned. Then there exist a function $K: \mathcal{C} \mapsto \mathbb{Z}$ and a measurable set $E$ such that

(5.1) $|E| \leq c\left(\frac{1}{\alpha} \sum \lambda_{Q}+\sum|S|\right)$

(5.2) $\left\{Q+2^{j} \operatorname{supp}(\mu)\right\} \subset E$ if $j<K(Q)$ and $Q \in \mathcal{C}$

(5.3) $\sigma(S(Q))<K(Q) \quad(Q \in \mathcal{C})$

(5.4) For each $\tau, \sigma \in \mathbb{Z}$ with $\sigma \leq \tau$, and any $q \in \Re_{\sigma}$

$$
\sum_{Q \subset q, K(Q) \leq \tau} \lambda_{Q} \leq 2^{n} \alpha 2^{(d-n) \sigma+n \tau} .
$$

Proof. The proof is a stopping-time argument controlled by two parameters $\tau$ and $\sigma$ as in the proof of Lemma 5.1 in [C2]. Let $m=\min \{\sigma(Q): Q \in \mathcal{C}\}$. Select an integer $\tau_{0}$ such that

$$
\tau_{0}>\max \{\sigma(Q): Q \in \mathcal{C}\}, \quad \sum_{Q \in \mathcal{C}} \lambda_{Q}<\alpha 2^{(d-n) m+n \tau_{0}} .
$$

For each fixed $\tau \in \mathbb{Z}$ with $\tau \leq \tau_{0}$, we define a sequence of functions $\Lambda_{\tau, \sigma}: \Re_{\sigma} \mapsto \mathbb{R}$ by a descending induction on $\sigma \in \mathbb{Z}$ with $\sigma \leq \tau$. And proceed with the same construction by a descending induction on $\tau$. At each step, we divide $\mathcal{C}$ into disjoint subcollections $\mathcal{C}_{1}$ and $\mathcal{C}_{2}$ which will increase as we proceed. Let $\mathcal{C}_{1}, \mathcal{C}_{2} \subset \mathcal{C}$ and $\tau \in \mathbb{Z}$ be fixed for the moment, and we define [Inner Loop] as

[Inner Loop] Define $\Lambda_{\tau, \sigma}: \Re_{\sigma} \mapsto \mathbb{R}$ with $\sigma \leq \tau$. For each $q \in \Re_{\sigma}$ define

$$
\Lambda_{\tau, \sigma}(q)=\sum_{Q \subset q ; Q \notin \mathcal{C}_{1} \cup \mathcal{C}_{2}} \lambda_{Q}
$$

First, begin with $\sigma=\tau$. If $\Lambda_{\tau, \sigma}(q)>\alpha 2^{(d-n) \sigma+n \tau}$ then we say that " $q$ is selected at step $(\tau, \sigma)$ " and put into $\mathcal{C}_{1}$ every $Q$ such that $Q \subset q$ and for such a $Q$ define $K(Q)=1+\tau$. Next replace $\sigma$ by $\sigma-1$ and repeat the process. Repeat until $\sigma<m$. Actually this part of process terminates once $\sigma$ is smaller than $m$. Finally, put into $\mathcal{C}_{2}$ every $Q \in \mathcal{C} \backslash \mathcal{C}_{1}$ such that $\sigma(Q) \geq \tau$ and for such a $Q$ define $K(Q)=1+\sigma(S(Q))$. Actually every $Q \in \mathcal{C} \backslash \mathcal{C}_{1} \cup \mathcal{C}_{2}$ satisfies $\sigma(Q) \leq \tau-1$.

Perform [Inner Loop] with $\mathcal{C}_{1}=\mathcal{C}_{2}=\emptyset$ and $\tau=\tau_{0}$. Next replace $\tau$ by $\tau-1$ and repeat [Inner Loop]. Repeat until $\tau=m-1$. After this process, we obtain $\mathcal{C}=\mathcal{C}_{1} \cup \mathcal{C}_{2}$, and clearly all selected $q$ are disjoint, and $K$ 
is well-defined. Note that there is the usual stopping-time condition

$$
\Lambda_{\tau, \sigma}(q) \leq 2^{n} \alpha 2^{(d-n) \sigma+n \tau}
$$

which holds for all $q \in \Re_{\sigma}$ when $\sigma \leq \tau \leq \tau_{0}$. This is because, if $\tau=\tau_{0}$ then the condition is clear from the initial condition on $\tau_{0}$. And when $\sigma \leq \tau<\tau_{0}$, suppose this fails. Then $\Lambda_{\tau+1, \sigma}(q) \geq \Lambda_{\tau, \sigma}(q)>\alpha 2^{(d-n) \sigma+n(\tau+1)}$. This means $q$ is selected at step $(\tau+1, \sigma)$, hence $\Lambda_{\tau, \sigma}(q)=0$ and we have contradiction.

Next we show (5.4), which says that for each $q \in \Re_{\sigma}$ with $\sigma \leq \tau$

$$
\sum_{Q \subset q ; K(Q) \leq \tau} \lambda_{Q} \leq 2^{n} \alpha 2^{(d-n) \sigma+n \tau} .
$$

When $\tau \geq \tau_{0}$, then the condition is clear from the initial condition of $\tau_{0}$. When $\tau \leq \tau_{0}$, then we note the fact that for each $q \in \Re_{\sigma}$ with $\sigma \leq \tau \leq \tau_{0}$

$$
\Lambda_{\tau, \sigma}(q)=\sum_{Q \subset q ; Q \notin \mathcal{C}_{1} \cup \mathcal{C}_{2}} \lambda_{Q} \geq \sum_{Q \subset q ; K(Q) \leq \tau} \lambda_{Q}
$$

Combining (6) and (7), we have (5.4) when $\sigma \leq \tau \leq \tau_{0}$. (7) will follow from the definition

$$
\Lambda_{\tau, \sigma}(q)=\sum_{Q \subset q ; Q \notin \mathcal{C}_{1} \cup \mathcal{C}_{2}} \lambda_{Q}
$$

and the fact that if $Q \in \mathcal{C}_{1} \cup \mathcal{C}_{2}$ at the beginning of step $(\tau, \sigma)$ then $K(Q)>\tau$. This is because, if $Q \in \mathcal{C}_{1}$ then $K(Q) \geq 1+\tau>\tau$, and if $Q \in \mathcal{C}_{2}$ then $K(Q)=1+\sigma(S(Q)) \geq 1+(1+\tau)>\tau$. Hence we have (5.4).

Next, we construct an exceptional set $E$. If $q$ is selected at step $(\tau, \sigma)$, then we define $\tau(q)=\tau$ and

$$
\begin{gathered}
T(q)=\bigcup_{j \leq \tau(q)+1}\left\{q+2^{j} \operatorname{supp}(\mu)\right\} \\
E=E_{1} \bigcup E_{2}, \quad E_{1}=\bigcup_{S \in \mathcal{S}} S^{*}, \quad E_{2}=\bigcup_{q: \text { selected }} T(q) .
\end{gathered}
$$

Thus we have

$$
\left|E_{1}\right| \leq c \sum|S|
$$

and

$$
\begin{aligned}
T(q) & =\bigcup_{j \leq \tau(q)+1}\left\{q+2^{j} \operatorname{supp}(\mu)\right\} \\
& =\bigcup_{j \leq \sigma(q)}\left\{q+2^{j} \operatorname{supp}(\mu)\right\} \bigcup_{\sigma(q)<j \leq \tau(q)+1}\left\{q+2^{j} \operatorname{supp}(\mu)\right\} .
\end{aligned}
$$

Because $\operatorname{supp}(\mu)$ is compact, if we regard $q^{*}$ as a proper expansion of $q$ then $\bigcup_{j<\sigma(q)}\left\{q+2^{j} \operatorname{supp}(\mu)\right\} \subset q^{*}$. And for $j>\sigma(q)$, if $x_{0}$ is the center 
of $q$, then by using translation invariance and dilation property of Lebesque measure, we have

$$
\begin{aligned}
\left|\left\{q+2^{j} \operatorname{supp}(\mu)\right\}\right| & \leq\left|\left\{B\left(x_{0}, 2^{\sigma(q)}\right)+2^{j} \operatorname{supp}(\mu)\right\}\right| \\
& =\left|\left\{B\left(0,2^{\sigma(q)}\right)+2^{j} \operatorname{supp}(\mu)\right\}\right| \\
& =2^{d j}\left|\left\{B\left(0,2^{\sigma(q)-j}\right)+\operatorname{supp}(\mu)\right\}\right| \\
& \leq c 2^{d j} 2^{d(\sigma(q)-j)} N\left(\operatorname{supp}(\mu), 2^{\sigma(q)-j}\right) \\
& \leq c 2^{(d-n) \sigma(q)+n j} .
\end{aligned}
$$

Hence

$$
|T(q)| \leq c\left(|q|+\sum_{\sigma(q)<j \leq \tau(q)+1} 2^{(d-n) \sigma(q)+n j}\right) \leq c 2^{(d-n) \sigma(q)+n \tau(q)}
$$

and we have

$$
\begin{aligned}
\left|E_{2}\right| & \leq \sum_{q: \text { selected }}|T(q)| \\
& \leq c \sum_{q: \text { selected }} 2^{(d-n) \sigma(q)+n \tau(q)} \\
& \leq \frac{c}{\alpha} \sum_{q: \text { selected }} \Lambda_{\tau, \sigma}(q) \\
& \leq \frac{c}{\alpha} \sum \lambda_{Q} .
\end{aligned}
$$

So we obtain (5.1). For (5.2), observe that if $Q \in \mathcal{C}_{1}$ then $Q$ belongs to some selected $q$, hence

$$
\bigcup_{j<K(Q)}\left\{Q+2^{j} \operatorname{supp}(\mu)\right\} \subset \bigcup_{j \leq K(Q)=\tau(q)+1}\left\{q+2^{j} \operatorname{supp}(\mu)\right\}=T(q) \subset E_{2}
$$

and if $Q \in \mathcal{C}_{2}$ then $Q$ belongs to some $S=S(Q) \in \mathcal{S}$, hence

$$
\bigcup_{j<K(Q)=1+\sigma(S(Q))}\left\{Q+2^{j} \operatorname{supp}(\mu)\right\} \subset S^{*} \subset E_{1}
$$

if we regard $S^{*}$ as a proper expansion of $S$. For (5.3), we replace $K$ by $K^{\prime}$ and define

$$
K(Q)=\max \left\{K^{\prime}(Q), 1+\sigma(S(Q))\right\} .
$$

Then (5.1) and (5.3) are satisfied. We must check (5.2) and (5.4). For (5.2), if $K(Q)=K^{\prime}(Q)$ then there is no problem. If $K(Q)=1+\sigma(S(Q))>j$ 
then the argument is the same as above. For (5.4)

$$
\sum_{Q \subset q ; K(Q) \leq \tau} \lambda_{Q} \leq \sum_{Q \subset q ; K^{\prime}(Q) \leq \tau} \lambda_{Q}
$$

and Lemma 5 follows.

\section{Proof of Theorem 1.}

Let $f \in H^{1}\left(\mathbb{R}^{d}\right)$ have the form of a finite sum

$$
f=\sum \lambda_{Q} a_{Q}
$$

where $\lambda_{Q}>0$ and $a_{Q}$, supported in $Q$, satifies

$$
\left\|a_{Q}\right\|_{L^{\infty}} \leq \frac{1}{|Q|}, \quad \int a_{Q}=0
$$

As was pointed out in [C2], a device of Garnett and Jones involving auxiliary dyadic grids allows us to assume that each $Q$ is dyadic. For $\alpha>0$, it is enough to show

$$
|\{x: \mathcal{M} f(x)>2 \alpha\}| \leq \frac{c}{\alpha} \sum \lambda_{Q} .
$$

Let $\mathcal{S}$ be as in Lemma 4 and define

$$
b=\sum_{S \in \mathcal{S}} \sum_{Q \subset S} \lambda_{Q} a_{Q}, \quad g=f-b .
$$

Then $\|g\|_{L^{\infty}} \leq \alpha$ from (4.3) and so $|\mathcal{M g}| \leq \alpha$ (by assuming $\mu$ has mass 1 ). Thus (8) will follow from

$$
|\{x: \mathcal{M} b(x)>\alpha\}| \leq \frac{c}{\alpha} \sum \lambda_{Q} .
$$

Let $\mathcal{S}$ be as above and $\mathcal{C}$ be the collection of $Q$ 's appearing in the definition of $b$. With $K$ and $E$ as in Lemma 5 , it is enough to prove

$$
\|\mathcal{M} b\|_{L^{2}\left(\mathbb{R}^{d} \backslash E\right)}^{2} \leq c \alpha \sum \lambda_{Q} .
$$

Let $\mu_{j}$ be the dilate of $\mu$ defined by

$$
\left\langle\phi, \mu_{j}\right\rangle=\int_{\mathbb{R}^{d}} \phi\left(2^{j} x\right) d \mu(x)
$$

then

$$
\mathcal{M} b(x)=\sup _{j \in \mathbb{Z}}\left|b * \mu_{j}(x)\right| .
$$


If $Q \in \mathcal{C}$, then by (5.3) $a_{Q} * \mu_{j}$ is supported in $E$ unless $j \geq K(Q)$. Thus for $x \notin E$, we have

$$
\begin{aligned}
|\mathcal{M} b(x)|^{2} & \leq \sum_{j}\left|b * \mu_{j}(x)\right|^{2} \\
& =\sum_{j}\left|\left(\sum_{K(Q) \leq j} \lambda_{Q} a_{Q}\right) * \mu_{j}(x)\right|^{2} \\
& =\sum_{j}\left|\sum_{s=0}^{\infty}\left(\sum_{K(Q)=j-s} \lambda_{Q} a_{Q}\right) * \mu_{j}(x)\right|^{2} .
\end{aligned}
$$

So for $x \notin E$, by Minkowski's inequality

$$
|\mathcal{M} b(x)| \leq \sum_{s=0}^{\infty}\left[\sum_{j}\left|\left(\sum_{K(Q)=j-s} \lambda_{Q} a_{Q}\right) * \mu_{j}(x)\right|^{2}\right]^{\frac{1}{2}} .
$$

Now (9) will follow from

$$
\left\|\left[\sum_{j}\left|\left(\sum_{K(Q)=j-s} \lambda_{Q} a_{Q}\right) * \mu_{j}\right|^{2}\right]^{\frac{1}{2}}\right\|_{L^{2}}^{2} \leq c(s+3) \alpha 2^{-\epsilon s} \sum \lambda_{Q}
$$

where $\epsilon=\min (1, n)$. And so from

$$
\left\|\left(\sum_{K(Q)=j-s} \lambda_{Q} a_{Q}\right) * \mu_{j}\right\|_{L^{2}}^{2} \leq c \alpha(s+3) 2^{-\epsilon s} \sum_{K(Q)=j-s} \lambda_{Q} .
$$

By scaling we may take $j=0$. And (10) will follow from

$$
\left\|\left(\sum_{K(Q)=-s} \lambda_{Q} a_{Q}\right) * \mu\right\|_{L^{2}}^{2} \leq c \alpha(s+3) 2^{-\epsilon s} \sum_{K(Q)=-s} \lambda_{Q} .
$$

Next as in Lemma 3 in $[\mathbf{O}]$, for each positive integer $N$, we define a sequence of functions $h_{N}$ and $L_{N}$. First we define $h_{N}$ by

$$
\hat{h}_{N}(\xi)=\frac{\chi_{|\xi| \leq N}(\xi)}{(1+|\xi|)^{n}} .
$$

Choose a radial function $\rho \in C_{c}^{\infty}\left(\mathbb{R}^{d}\right)$ such that

$$
\int \rho=1, \quad \operatorname{supp}(\rho) \subset[-1,1]^{d}, \quad \hat{\rho} \geq 0 .
$$


Now let $L_{N}=\rho h_{N}$ and

$$
\hat{L}(\xi)=\lim _{N \rightarrow \infty} \hat{L}_{N}(\xi)=\int \frac{\hat{\rho}(y) d y}{(1+|\xi-y|)^{n}} .
$$

Lemma 6. We have the following:

(6.1) $\operatorname{supp}\left(L_{N}\right) \subset[-1,1]^{d}$

(6.2) $\hat{L}_{N}(\xi) \geq \frac{c}{(1+|\xi|)^{n}} \quad$ if $|\xi| \leq N-1$

(6.3) For each $\beta$, we have

$$
\left|\partial_{\xi}^{\beta} \hat{L}(\xi)\right| \leq \frac{A_{\beta}}{(1+|\xi|)^{n+|\beta|}} .
$$

Proof. It is easy to check (6.1), (6.2). For (6.3), first we assume $d \geq 2$, then we have

$$
\begin{aligned}
\left|\partial_{\xi}^{\beta} \hat{L}(\xi)\right| & =\left|\int \hat{\rho}(y) \partial_{\xi}^{\beta} \frac{1}{(1+|\xi-y|)^{n}} d y\right| \\
& \leq c \int \frac{|\hat{\rho}(y)| d y}{(1+|\xi-y|)^{n+|\beta|}} \leq \frac{c}{(1+|\xi|)^{n+|\beta|}} .
\end{aligned}
$$

When $d=1$, we use

$$
\hat{L}(\xi)=\int_{\xi}^{\infty} \frac{\hat{\rho}(y) d y}{(1+y-\xi)^{n}}+\int_{-\infty}^{\xi} \frac{\hat{\rho}(y) d y}{(1+\xi-y)^{n}}
$$

and do similarly as before.

Next, let $\phi_{N}$ be the inverse Fourier transform of $\left(\hat{L}_{N}\right)^{\frac{1}{2}}$, then $L_{N}=$ $\phi_{N} * \widetilde{\phi}_{N}$. And we have

$$
\left|\hat{\phi}_{N}(\xi)\right|^{2} \geq \frac{c}{(1+|\xi|)^{n}} \quad \text { when }|\xi| \leq N-1 .
$$

Therefore, returning to (11) we have

$$
\begin{aligned}
\left\|\left(\sum_{K(Q)=-s} \lambda_{Q} a_{Q}\right) * \mu\right\|_{L^{2}}^{2} & =c \int\left|\left(\sum_{K(Q)=-s} \lambda_{Q} \hat{a}_{Q}\right)(\xi)\right|^{2}|\hat{\mu}(\xi)|^{2} d \xi \\
& \leq c \int\left|\left(\sum_{K(Q)=-s} \lambda_{Q} \hat{a}_{Q}\right)(\xi)\right|^{2} \liminf _{N \rightarrow \infty}\left|\hat{\phi}_{N}(\xi)\right|^{2} d \xi \\
& \leq c \liminf _{N \rightarrow \infty} \int\left|\left(\sum_{K(Q)=-s} \lambda_{Q} \hat{a}_{Q}\right)(\xi)\right|^{2}\left|\hat{\phi}_{N}(\xi)\right|^{2} d \xi \\
& \leq c \liminf _{N \rightarrow \infty}\left\|\left(\sum_{K(Q)=-s} \lambda_{Q} a_{Q}\right) * \phi_{N}\right\|_{L^{2}}^{2} .
\end{aligned}
$$


So (11) will follow from

$$
\begin{aligned}
\liminf _{N \rightarrow \infty} & \left\|\left(\sum_{K(Q)=-s} \lambda_{Q} a_{Q}\right) * \phi_{N}\right\|_{L^{2}}^{2} \\
& \leq c \alpha(s+3) 2^{-\epsilon s} \sum_{K(Q)=-s} \lambda_{Q} .
\end{aligned}
$$

Because $\operatorname{supp}\left(L_{N}\right) \subset[-1,1]^{d}$, and for each $Q, Q^{\prime} \in \mathcal{C}$ such that $K(Q)=K\left(Q^{\prime}\right)=-s$, we have $\sigma(Q), \sigma\left(Q^{\prime}\right) \leq K(Q)=K\left(Q^{\prime}\right)=-s$, hence $\left|\left\langle a_{Q^{\prime}} * L_{N}, a_{Q}\right\rangle\right|=0$ when $\operatorname{dist}\left(Q, Q^{\prime}\right)>4$. So we have

$$
\begin{aligned}
& \liminf _{N \rightarrow \infty}\left\|\left(\sum_{K(Q)=-s} \lambda_{Q} a_{Q}\right) * \phi_{N}\right\|_{L^{2}}^{2} \\
& \leq 2 \liminf _{N \rightarrow \infty} \sum_{Q, Q^{\prime} ; \sigma\left(Q^{\prime}\right) \geq \sigma(Q)} \lambda_{Q} \lambda_{Q^{\prime}}\left|\left\langle a_{Q^{\prime}} * L_{N}, a_{Q}\right\rangle\right| \\
& \operatorname{dist}\left(Q, Q^{\prime}\right) \leq 4 \\
& \leq 2 \liminf _{N \rightarrow \infty} \sum_{\begin{array}{r}
Q, Q^{\prime}, \sigma\left(Q^{\prime}\right) \geq \sigma(Q) \\
\operatorname{dist}\left(Q, Q^{\prime}\right) \leq 4
\end{array}} \lambda_{Q} \lambda_{Q^{\prime}}\left|\left\langle\hat{a}_{Q^{\prime}} \hat{L}_{N}, \hat{a}_{Q}\right\rangle\right| \\
& \leq 2 \sum_{Q^{\prime}} \sum_{Q \subset Q^{\prime *}} \lambda_{Q} \lambda_{Q^{\prime}}\left|\left\langle\hat{a}_{Q^{\prime}} \hat{L}, \hat{a}_{Q}\right\rangle\right| \\
& \operatorname{dist}\left(Q, Q^{\prime}\right) \leq 4 \\
& +2 \sum_{Q^{\prime}} \sum_{\begin{array}{c}
Q \cap Q^{\prime *}=\emptyset \\
\operatorname{dist}\left(Q, Q^{\prime}\right) \leq 4
\end{array}} \lambda_{Q} \lambda_{Q^{\prime}}\left|\left\langle\hat{a}_{Q^{\prime}} \hat{L}, \hat{a}_{Q}\right\rangle\right| \\
& =\mathrm{I}+\mathrm{II} \text {. }
\end{aligned}
$$

Lemma 7. We have the following:

$$
\begin{aligned}
& \left|\left\langle\hat{a}_{Q^{\prime}} \hat{L}, \hat{a}_{Q}\right\rangle\right| \leq c 2^{-(d-n) \sigma\left(Q^{\prime}\right)} \\
& \left\langle\hat{a}_{Q^{\prime}} \hat{L}, \hat{a}_{Q}\right\rangle \mid \leq c \frac{2^{\sigma(Q)}}{\left(\operatorname{dist}\left(Q, Q^{\prime}\right)\right)^{d-n+1}} \quad \text { when } Q \bigcap Q^{\prime *}=\emptyset
\end{aligned}
$$

Proof. For (7.1), we consider as two cases; $d=n$ and $d>n$. When $d=n$, we use the easy estimates.

$$
\left|\hat{a}_{Q}(\xi)\right| \leq c \min \left(1,|\xi| 2^{\sigma(Q)}\right), \quad\left\|\hat{a}_{Q}\right\|_{L^{2}}^{2} \leq c 2^{-d \sigma(Q)} .
$$


Hence we have

$$
\begin{aligned}
\left|\left\langle\hat{a}_{Q^{\prime}} \hat{L}, \hat{a}_{Q}\right\rangle\right| \leq & \left\|\hat{a}_{Q}\right\|_{L^{\infty}} \int \frac{\left|\hat{a}_{Q^{\prime}}(\xi)\right|}{(1+|\xi|)^{d}} d \xi \\
\leq & c\left(\int_{|\xi|<2^{-\sigma\left(Q^{\prime}\right)}} \frac{|\xi| 2^{\sigma\left(Q^{\prime}\right)}}{(1+|\xi|)^{d}} d \xi\right. \\
& \left.\quad+\left\|\hat{a}_{Q^{\prime}}\right\|_{L^{2}}\left[\int_{|\xi| \geq 2^{-\sigma\left(Q^{\prime}\right)}}(1+|\xi|)^{-2 d} d \xi\right]^{1 / 2}\right) \\
\leq & c .
\end{aligned}
$$

When $d>n$, choose $\eta \in C_{c}^{\infty}\left(\mathbb{R}^{d}\right)$ such that $\eta(\xi)=1$ for $|\xi| \leq 1$, and $\eta(\xi)=0$ for $|\xi| \geq 2$. Define another function $\delta$ by $\delta(\xi)=\eta(\xi)-\eta(2 \xi)$. Then we have

$$
1=\eta(\xi)+\sum_{j=1}^{\infty} \delta\left(2^{-j} \xi\right), \text { for all } \xi
$$

and

$$
\hat{L}(\xi)=\eta(\xi) \hat{L}(\xi)+\sum_{j=1}^{\infty} \hat{L}(\xi) \delta\left(2^{-j} \xi\right)=m_{0}(\xi)+\sum_{j=1}^{\infty} m_{j}(\xi)
$$

We set

$$
K_{j}(x)=\int e^{2 \pi i x \cdot \xi} m_{j}(\xi) d \xi
$$

Observe that

$$
\left|(-2 \pi i x)^{\gamma} \partial_{x}^{\beta} K_{j}(x)\right|=\left|\int \partial_{\xi}^{\gamma}\left[(2 \pi i \xi)^{\beta} m_{j}(\xi)\right] e^{2 \pi i x \cdot \xi} d \xi\right| .
$$

By (6.3) and support condition of the integrand, we can show

$$
\left|x^{\gamma} \partial_{x}^{\beta} K_{j}(x)\right| \leq A_{\gamma, \beta} 2^{j(d-n+|\beta|-|\gamma|)} .
$$

Hence, for each positive integer $M$, we have

$$
\left|\partial_{x}^{\beta} K_{j}(x)\right| \leq A_{M, \beta}|x|^{-M} 2^{j(d-n+|\beta|-M)},
$$

and so

$$
\sum_{j=0}^{\infty}\left|\partial_{x}^{\beta} K_{j}(x)\right|=\sum_{2^{j} \leq|x|^{-1}}+\sum_{2^{j}>|x|^{-1}} .
$$

First with $M=0$, we have

$$
\begin{aligned}
\sum_{2^{j} \leq|x|^{-1}}\left|\partial_{x}^{\beta} K_{j}(x)\right| & \leq A_{\beta} \sum_{2^{j} \leq|x|^{-1}} 2^{j(d-n+|\beta|)} \\
& \leq A_{\beta}^{\prime}|x|^{-d+n-|\beta|} .
\end{aligned}
$$


Second with $M>d-n+|\beta|$, we have

$$
\begin{aligned}
\sum_{2^{j}>|x|^{-1}}\left|\partial_{x}^{\beta} K_{j}(x)\right| & \leq A_{\beta} \sum_{2^{j}>|x|^{-1}}|x|^{-M} 2^{j(d-n+|\beta|-M)} \\
& \leq A_{\beta}^{\prime}|x|^{-d+n-|\beta|} .
\end{aligned}
$$

Hence we have

$$
\sum_{j=0}^{\infty}\left|\partial_{x}^{\beta} K_{j}(x)\right| \leq A_{\beta}^{\prime}|x|^{-d+n-|\beta|}
$$

Returning to (7.1), by Lebesgue Dominated Convergence Theorem, we have

$$
\begin{aligned}
\left|\left\langle\hat{a}_{Q} \hat{L}, \hat{a}_{Q^{\prime}}\right\rangle\right| & =\left|\sum_{j=0}^{\infty}\left\langle\hat{a}_{Q^{\prime}} m_{j}, \hat{a}_{Q}\right\rangle\right| \\
& =\left|\sum_{j=0}^{\infty}\left\langle a_{Q^{\prime}} * K_{j}, a_{Q}\right\rangle\right| \\
& \leq\left\langle\left|a_{Q^{\prime}}\right| * \sum_{j=0}^{\infty}\left|K_{j}\right|,\left|a_{Q}\right|\right\rangle \\
& \leq\left\|a_{Q}\right\|_{L^{1}} \sup _{x \in Q}\left|a_{Q^{\prime}}\right| *\left(\sum_{j=0}^{\infty}\left|K_{j}(x)\right|\right) \\
& \leq c\left\|a_{Q^{\prime}}\right\|_{L^{\infty}} \sup _{x \in Q} \int_{Q^{\prime}} \sum_{j=0}^{\infty}\left|K_{j}(x-y)\right| d y
\end{aligned}
$$

and by (14), we have

$$
\sup _{x \in Q} \int_{Q^{\prime}} \sum_{j=0}^{\infty}\left|K_{j}(x-y)\right| d y \leq c \sup _{x \in Q} \int_{Q^{\prime}}|x-y|^{-d+n} d y \leq c 2^{n \sigma\left(Q^{\prime}\right)}
$$

when $d>n$. Hence when $d>n$, we have

$$
\left|\left\langle\hat{a}_{Q^{\prime}} \hat{L}, \hat{a}_{Q}\right\rangle\right| \leq c 2^{-(d-n) \sigma\left(Q^{\prime}\right)},
$$


and obtain (7.1). For (7.2), let $\widetilde{x}$ be the center of $Q$, then

$$
\begin{aligned}
& \left|\left\langle\hat{a}_{Q^{\prime}} \hat{L}, \hat{a}_{Q}\right\rangle\right| \\
& =\left|\sum_{j=0}^{\infty}\left\langle a_{Q^{\prime}} * K_{j}, a_{Q}\right\rangle\right| \\
& =\left|\sum_{j=0}^{\infty} \iint a_{Q^{\prime}}(y)\left(K_{j}(x-y)-K_{j}(\widetilde{x}-y)\right) a_{Q}(x) d x d y\right| \\
& \leq \iint\left|a_{Q^{\prime}}(y)\right|\left|a_{Q}(x)\right| \sum_{j=0}^{\infty}\left|\left(K_{j}(x-y)-K_{j}(\widetilde{x}-y)\right)\right| d x d y \\
& \leq \iint\left|a_{Q^{\prime}}(y)\right|\left|a_{Q}(x)\right| \sum_{j=0}^{\infty}\left|x-\widetilde{x} \| \nabla K_{j}\left(\widetilde{x}_{j}-y\right)\right| d x d y,
\end{aligned}
$$

where $\widetilde{x}_{j}$ lies in the line connecting $\widetilde{x}$ and $x$. By (13), for each positive integer $M$, we have

$$
\begin{aligned}
\left|\nabla K_{j}\left(\widetilde{x}_{j}-y\right)\right| & \leq A_{M}\left|\widetilde{x}_{j}-y\right|^{-M} 2^{j(d-n+1-M)} \\
& \leq A_{M}^{\prime} \operatorname{dist}\left(Q, Q^{\prime}\right)^{-M_{2}} 2^{j(d-n+1-M)},
\end{aligned}
$$

when $Q \bigcap Q^{\prime *}=\emptyset$. Hence, by the same method as in (14), we have

$$
\sum_{j=0}^{\infty}\left|\nabla K_{j}\left(\widetilde{x}_{j}-y\right)\right| \leq c\left(\operatorname{dist}\left(Q, Q^{\prime}\right)\right)^{-d+n-1} \text { when } Q \bigcap Q^{\prime *}=\emptyset
$$

And so we have

$$
\begin{aligned}
\left|\left\langle\hat{a}_{Q^{\prime}} \hat{L}, \hat{a}_{Q}\right\rangle\right| & \leq c \frac{2^{\sigma(Q)}}{\left(\operatorname{dist}\left(Q, Q^{\prime}\right)\right)^{d-n+1}} \iint\left|a_{Q^{\prime}}(y)\right|\left|a_{Q}(x)\right| d x d y \\
& \leq c \frac{2^{\sigma(Q)}}{\left(\operatorname{dist}\left(Q, Q^{\prime}\right)\right)^{d-n+1}}
\end{aligned}
$$

when $Q \bigcap Q^{\prime *}=\emptyset$.

- Estimation of part $I$ : 

have

By (5.4) we have $\sum_{Q \subset Q^{\prime *}} \lambda_{Q} \leq c \alpha 2^{(d-n) \sigma\left(Q^{\prime}\right)-n s}$ and use (7.1). So we

$$
\begin{aligned}
I & \leq c \sum_{Q^{\prime}} \sum_{Q \subset Q^{\prime *}} \lambda_{Q} \lambda_{Q^{\prime}} 2^{-(d-n) \sigma\left(Q^{\prime}\right)} \\
& \leq c\left(\sum_{Q^{\prime}} \lambda_{Q^{\prime}} 2^{-(d-n) \sigma\left(Q^{\prime}\right)}\right)\left(\alpha 2^{(d-n) \sigma\left(Q^{\prime}\right)-n s}\right) \\
& \leq c 2^{-n s} \alpha \sum_{K(Q)=-s} \lambda_{Q} .
\end{aligned}
$$

- Estimation of part $I I$ :

If $Q \cap Q^{\prime *}=\emptyset$, then by $(7.2)$ and $\sigma(Q) \leq \sigma\left(Q^{\prime}\right)$, we have

$$
\begin{aligned}
& I I \leq c \sum_{Q^{\prime}} \sum_{Q \cap Q^{\prime *}=\emptyset} \lambda_{Q} \lambda_{Q^{\prime}} \frac{2^{\sigma\left(Q^{\prime}\right)}}{\operatorname{dist}\left(Q, Q^{\prime}\right)^{(d-n)+1}} \\
& \operatorname{dist}\left(Q, Q^{\prime}\right) \leq 4 \\
& \leq c\left(\sum_{Q^{\prime}} 2^{\sigma\left(Q^{\prime}\right)} \lambda_{Q^{\prime}}\right)\left(\sum_{Q \cap Q^{\prime *}=\emptyset} \frac{\lambda_{Q}}{\operatorname{dist}\left(Q, Q^{\prime}\right)^{(d-n)+1}}\right) \\
& \leq c\left(\sum_{Q^{\prime}} 2^{\sigma\left(Q^{\prime}\right)} \lambda_{Q^{\prime}}\right)\left(\sum_{\substack{Q ; \operatorname{dist}\left(Q, Q^{\prime}\right) \sim 2^{m+\sigma\left(Q^{\prime}\right)} \\
m+\sigma\left(Q^{\prime}\right) \leq-s+2}}+\sum_{\substack{Q ; \operatorname{dist}\left(Q, Q^{\prime}\right) \sim 2^{m+\sigma\left(Q^{\prime}\right)} \\
-s+3 \leq m+\sigma\left(Q^{\prime}\right) \leq 2}}\right) \\
& \leq c\left(\sum_{Q^{\prime}} 2^{\sigma\left(Q^{\prime}\right)} \lambda_{Q^{\prime}}\right)\left(I I_{1}+I I_{2}\right) \text {. }
\end{aligned}
$$

For each positive integer $m$, consider the contribution of all $\lambda_{Q}$ over all $Q$ disjoint from $Q^{\prime *}$ with $\sigma(Q) \leq \sigma\left(Q^{\prime}\right)$. So we have $\operatorname{dist}\left(Q, Q^{\prime}\right) \sim 2^{m+\sigma\left(Q^{\prime}\right)}$. All such $Q$ are contained in the union of a fixed number of elements of $\Re_{m+\sigma\left(Q^{\prime}\right)}$. Hence when $m+\sigma\left(Q^{\prime}\right) \leq-s+2$, we can use (5.4) to obtain

$$
\begin{aligned}
I I_{1} & =\sum_{\substack{Q ; \operatorname{dist}\left(Q, Q^{\prime}\right) \sim^{m+\sigma\left(Q^{\prime}\right)} \\
m+\sigma\left(Q^{\prime}\right) \leq-s+2}} \frac{\lambda_{Q}}{\operatorname{dist}\left(Q, Q^{\prime}\right)^{(d-n)+1}} \\
& \leq c \sum_{m \geq 0} \alpha 2^{-(d-n+1)\left(m+\sigma\left(Q^{\prime}\right)\right)} 2^{(d-n)\left(m+\sigma\left(Q^{\prime}\right)\right)-n s} \\
& \leq c \alpha 2^{-\sigma\left(Q^{\prime}\right)} 2^{-n s} .
\end{aligned}
$$


Next, consider all $Q$ with $\operatorname{dist}\left(Q, Q^{\prime}\right) \sim 2^{m+\sigma\left(Q^{\prime}\right)}$ and $m+\sigma\left(Q^{\prime}\right) \geq-s+3$. Recall that each $Q \in C$ is contained in $S(Q)$ for some $S(Q) \in \mathcal{S}$. Since $K(Q)=-s$ and $K(Q)>\sigma(S(Q))$, we obtain $\operatorname{dist}\left(S(Q), Q^{\prime}\right) \geq 2^{-s}$. Also, by (4.1), we have $\sum_{Q \subset S} \lambda_{Q} \leq c \alpha|S|$ for every $S \in \mathcal{S}$, hence we obtain

$$
\begin{aligned}
I I_{2} & =\sum_{\substack{Q ; \operatorname{dist}\left(Q, Q^{\prime}\right) \sim 2^{m+\sigma\left(Q^{\prime}\right)} \\
-s+3 \leq m+\sigma\left(Q^{\prime}\right) \leq 2}} \frac{\lambda_{Q}}{\operatorname{dist}\left(Q, Q^{\prime}\right)^{(d-n)+1}} \\
& \leq c \sum \frac{\lambda_{Q}}{\operatorname{dist}\left(S(Q), Q^{\prime}\right)^{(d-n)+1}} \\
& \leq c \alpha \sum \frac{|S|}{\operatorname{dist}\left(S, Q^{\prime}\right)^{(d-n)+1}} \\
& \leq c \alpha \int_{2^{-s} \leq|y| \leq 4}^{|y|^{-(d-n+1)} d y} \\
& \leq c \alpha\left(s 2^{(1-n) s}+1\right) .
\end{aligned}
$$

Finally, since $\sigma\left(Q^{\prime}\right)<K\left(Q^{\prime}\right)=-s$, we obtain

$$
\begin{aligned}
I I & \leq c \sum_{Q^{\prime}} 2^{\sigma\left(Q^{\prime}\right)}\left(\alpha 2^{-\sigma\left(Q^{\prime}\right)} 2^{-n s}+\alpha\left(s 2^{(1-n) s}+1\right)\right) \lambda_{Q^{\prime}} \\
& \leq c(s+3) \alpha 2^{-\epsilon s} \sum_{K(Q)=-s} \lambda_{Q}
\end{aligned}
$$

where $\epsilon=\min (n, 1)$. This completes the proof of (12) and Theorem 1.

Acknowledgements. The results of this paper were obtained during my Ph.D. studies at Pohang University of Science and Technology and will be a part of my thesis. I would like to express deep gratitude to my advisor Jong-Guk Bak for guidance and support.

\section{References}

[Ca] A.P. Calderón, An atomic decomposition of distribution in $H^{p}$ space, Adv. in Math., 25 (1977), 216-225, MR 56 \#6376, Zbl 379.46050.

[C1] M. Christ, Hilbert transforms along curves, I: Nilpotent groups, Ann. of Math., 122 (1985), 575-596, MR 87f:42039a, Zbl 593.43011.

[C2] Weak type $(1,1)$ bounds for rough operators, Ann. of Math., 128 (1988), 19-42, MR 89m:42013, Zbl 666.47027.

[C3] , Restriction of Fourier transform to submanifolds of low codimension, Ph.D. Thesis, University of Chicago, 1982.

[CDMM] M. Cowling, S. Disney, G. Mauceri and D. Müller, Damping oscillatory integrals, Invent. Math., 101 (1990), 237-260, MR 91d:58242, Zbl 733.42011. 
[CM] M. Cowling and G. Mauceri, Oscillatory integrals and Fourier transforms of surface carried measures, Trans. Amer. Math. Soc., 304 (1987), 53-68, MR 89e:42013, Zbl 647.42009.

[DR] J. Duoandikoetxea and J. L. Rubio de Francia, Maximal and singular integral operators via Fourier transform estimates, Invent. Math., 84 (1986), 541-561, MR 87f:42046, Zbl 568.42012.

[F] H. Federer, Geometric Measure Theory, Springer-Verlag, 1969, MR 41 \#1976, Zbl 176.00801.

[Ka] J.P. Kahane, Some Random Series of Functions, Cambridge University Press, 1986, MR 87m:60119.

[Kö] T. Körner, On a theorem of Ivasev-Musatov III, Proc. London Math. Soc., 53(1) (1986), 143-192.

[L] W. Littman, Fourier transforms of surface carried measures, Bull. Amer. Math. Soc., 69 (1963), 776-770, MR 27 \#5086, Zbl 143.34701.

[O] D.M. Oberlin, An endpoint estimate for some maximal operators, Revista Mat. Iberoamerica, 12(3) (1996), 641-652, MR 97m:42016, Zbl 871.42015.

[SW] E.M. Stein and G. Weiss, Introduction to Fourier Analysis on Euclidean Spaces, Princeton University Press, 1971, MR 46 \#4102, Zbl 232.42007.

Received January 10, 2000.

Department of Mathematics

Pohang University of Science And Technology

POHANG 790-784

KoreA

E-mail address: yaryong@hanmail.net 